\title{
Recall memory for visually presented chess positions
}

\author{
PETER W. FREY and PETER ADESMAN \\ Northwesterm University, Evanston, Ilinois 60201
}

\begin{abstract}
A series of three experiments replicated and extended earlier research reported by Chase and Simon (1973), de Groot (1965), and Charness (Note 1). The first experiment demonstrated that the relationship between memory for chess positions and chess skill varies directly with the amount of chess-specific information in the stimulus display. The second experiment employed tachistoscopic displays to incrementally "build" tournament chess positions by meaningful or nonmeaningful chunks and demonstrated that meaningful piece groupings during presentation markedly enhance subsequent recall performance. The third experiment tested memory for one of two positions presented in immediate sequence and demonstrated that explanations based on a limited-capacity short-term memory (Chase \& Simon, 1973) are not adequate for explaining performance on this memory task.
\end{abstract}

The game of chess provides a useful working environment for the analysis of specialized informationprocessing skills. Skill in chess is acquired only with extensive exposure to the game. From an analysis of verbal protocols, de Groot (1965) established that chess masters and less able players use similar thought processes in analyzing a complex chess position. They consider a similar number of moves (about 35), calculate to similar depths (about 7 plies), make the same number of fresh starts (about 7), and analyze a similar number of moves per minute (about 3 ). The only major difference de Groot noted was that the masters invariably analyzed stronger moves than the weaker players. This conclusion (i.e., better players are better because they select better moves) was not terribly illuminating.

de Groot's (1965) research did indicate that masters differed from weaker players in their ability to recall a chess position from an unfamiliar game after it had been presented for only $5 \mathrm{sec}$. Masters recalled $93 \%$ of 22 pieces, while strong club players recalled about $51 \%$. Chase and Simon (1973) have shown that this recall ability is chess specific by replicating de Groot's result and including a control condition in which the chess pieces were arranged randomly. With a 5 -sec exposure of quiet middle-game positions, their master recalled $81 \%$ of the 22 pieces, while a novice recalled only $33 \%$. With the randomized positions, all subjects recalled only three to five pieces correctly. This result indicates that the master and novice have similar visual memory capacities for nonmeaningful piece configurations. Thus, the ability to play chess well seems to depend on a

The design and interpretation of these experiments benefited greatly from discussions with Neil Charness, Eliot Hearst, Henry Helff, Michael Humphreys, and Benton J. Underwood. The authors thank Stanton Tripodis for data collection assistance in Experiment I. Reprint requests should be addressed to Peter Frey, Department of Psychology, Northwestern University, Evanston, Illinois 60201. learned perceptual skill rather than on the acquisition of a sophisticated problem-solving strategy.

\section{EXPERIMENT I}

Our first study attempted to replicate and extend the findings of de Groot (1965) and of Chase and Simon (1973) using chess diagrams which were projected on a ground-glass screen in an experimental chamber. The degree of chess-specific information in the displays was manipulated by presenting randomized positions and meaningful positions, as was done by Chase and Simon (1973), and by including, in addition, entire game segments presented in a move-by-move sequence. It is unusual for an experienced chess player to encounter a middle-game position "without any knowledge of the move sequence which led to that position." Games are generally played and analyzed as wholes rather than as isolated positions. The third condition in our experiment was an attempt to present a meaningful chess position in the context of the move sequence which led to its development. Our expectation was that recall in this condition would covary more strongly with chess skill than recall in the other two conditions.

\section{Method}

Subjects. The subjects were 13 male volunteers from the Northwestern University chess club. Each subject was assigned to one of three groups based on his rating from the United States Chess Federation.

Apparatus. The subjects were seated in a sound-attenuated IAC booth (Industrial Acoustics Corporation, Bronx, New York) that had a ground-glass screen on one wall. The stimulus displays (35-mm photographic slides) were projected on this screen from the exterior. The duration of each slide was controlled by a two-channel projection tachistoscope (Ralph Gerbrands Company, Model 61170). The displays were photographs of chess diagrams available from the United States Chess Federation. The subject sat in the booth facing the ground-glass screen.

Procedure. The experimental design was a 3 by 3 factorial, with chess skill as a between-subject factor and type of display 


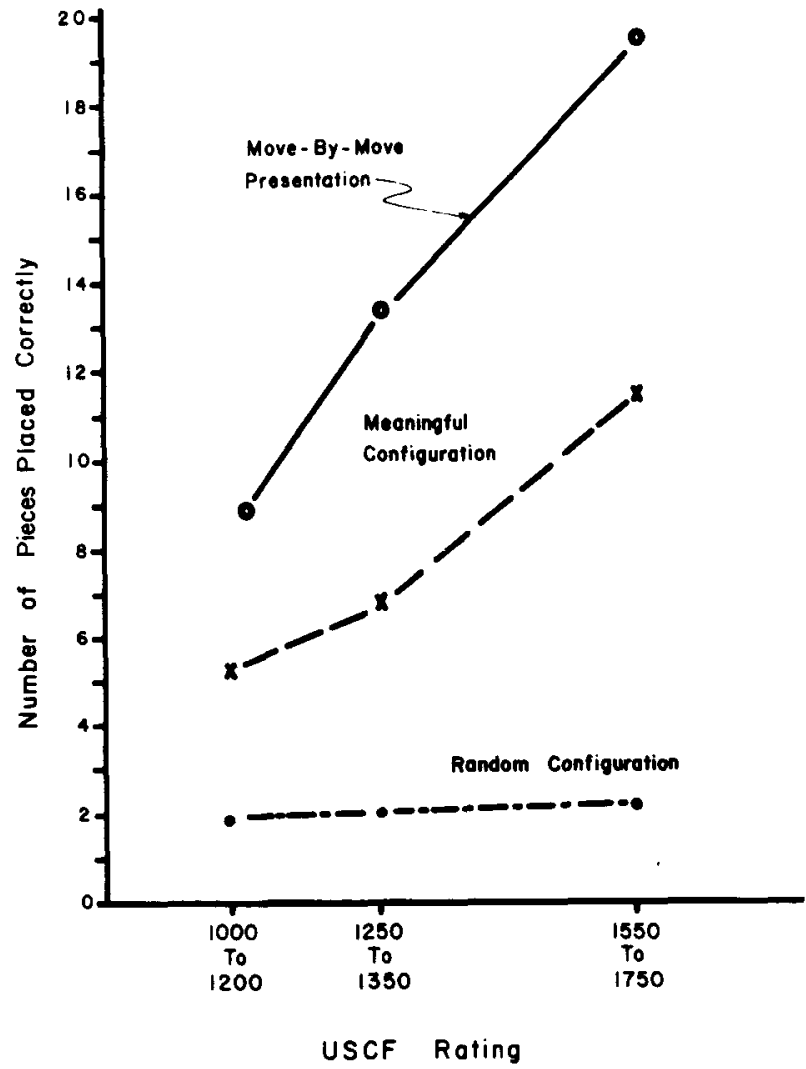

Figure 1. Number of pieces placed correctly as a function of chess skill and presentation condition in Experiment $I$.

as a within-subject factor. Ihe positions to be recalled were quiet middle-game positions which arose in tournament games after 22 moves or a randomized transformation of one of these positions. In each case, the position involved 23 or 24 pieces. In two of the conditions (random and meaningful), the positions were displayed only once for $8 \mathrm{sec}$. In the move-by-move condition, each sequence started with the standard beginning configuration for chess and then sequenced, move-by-move, through the first 44 positions ( 22 moves for each side) at $2 \mathrm{sec} /$ position ( $88 \mathrm{sec}$ total presentation time). The final position, which was the subject of the recall test, appeared for only $2 \mathrm{sec}$, in the same way as the other positions in the sequence. Each subject received 4 trials in each condition and, thus, 12 trials total. The order of trials was the same for all subjects. Each condition occurred once during Trials 1-3, once again during Trials 4-6, once again during Trials $7-9$, etc.

The subjects were told that chess positions would be projected on the ground-glass screen and that the display would sometimes involve a series of positions in sequence. Each was then instructed to attend carefully to the screen during each trial and to start reconstructing the last position only after the screen became "blank." After each trial, the subject was given $90 \mathrm{sec}$ to reconstruct the position using a chess set which had been placed on a table in the IAC booth. The experimenter entered the booth after $30 \mathrm{sec}$ of the recall periods and recorded the subject's piece placements.

\section{Results}

The recall performance of the subjects was measured by the number of pieces placed correctly for each position. The average recall scores across the four positions in each condition are presented in Figure 1. There were statistically significant effects of both chess Skill $[F(2,10)=14.45, p<.001]$ and Display conditions $[F(2,20)=109.52, p<.001]$. The interaction of these two factors was also significant $[F(4,20)=7.52$ $\mathrm{p}<.001]$. The standard error of the means for the between-subject variable (Skill level) was 2.83 pieces. The standard error of the means for the within-subject variable (Display condition) was 2.16 pieces. The major hypothesis we wished to test was that the effect of chess Skill would differ as a function of the Display condi. tions. Our prediction was that the slope of the function relating recall performance to chess Skill would become increasingly steep as the Display condition involved more chess-specific information. A test for trend (Myers. 1972 , p. 368) indicated that the interaction was primarily due to differences in the linear components $[F(2,30)=11.78, p<.001]$. Subsequent analyses com. paring slopes under the individual conditions indicated that the random condition had a different slope than the meaningful condition $[F(1,30)=8.37, p<.01]$ and that the slopes in the meaningful condition and the move-by. move condition just missed being statistically significant $[F(1,30)=3.73, \mathrm{p}<.07]$.

\section{Discussion}

The first experiment provides a clear replication of de Groot's (1965) finding that recall of briefly exposed meaningful chess configurations varies directly with the chess skill of the subject, and also replicates Chase and Simon's (1973) finding that this outcome is not ob. served with random piece configurations. The results demonstrate that these prior findings can still be ob. tained when the chess skill factor is manipulated in a restricted range. Our best subjects were far less skillful than the masters employed by de Groot and by Chase and Simon. In addition, our results generalize the previous methodology to chess diagrams which are presented using a projection procedure. The steeper slope of the recall function in the move-by-move condition provides a further demonstration that the amount of chess-specific information is the key factor for producing superior recall of chess positions by skilled players.

\section{EXPERIMENT II}

Chase and Simon (1973) presented latency data from their memory task and from a reconstruction task which indicated that pieces placed with a short interpieceplacement latency tend to be closely related in a chessical sense. Their interpretation of this clustering phenomena was that each piece grouping was a perceptual chunk which had a specific, possibly nonverbal, label. Charness (Note 1) presented verbal descriptions of chess piece configurations by calling out the individual pieces and their location one square at a time. His data indicated that the order of presentation was important in that "meaningful" orderings led to better recall than presentation by columns. Charness concluded that presentation in meaningful perceptual clusters strongly aided the subjects in visualizing the positions.

Our second experiment replicated Charness' (Note 1) 
rocedure, except that a visual manipulation of the legree of meaningful clustering was used. This manipulaion was implemented by tachistoscopically presenting $\downarrow$ position in six segments in which the position "dereloped" in an incremental fashion by either meaningful or nonmeaningful chunks. The expectation, based on Tharness' findings, was that recall performance would be setter when the information was presented in meaningul chunks.

\section{Method}

Subjects. The subjects were 27 volunteers from the Evanston shess club and the Northwestern University chess club. Each subject was assigned to one of three skill levels. The nine highskill subjects had USCF ratings between 1,800 and 2,000, the nine medium-skill subjects had ratings between 1,400 and 1,600 , and the nine low-skill subjects were novices without a USCF rating or with a rating below 1,200 . The subjects ranged in age from 17 years to 48 years and were predominantly male (i.e., only one female).

Apparatus. The equipment and data collection arrangement were the same as in the first experiment. Because some of our novice chess players in Experiment I seemed to be unfamiliar with the chess diagrams, our displays for the second experiment were made by photographing an actual chess set from the front and slightly above and to the side.

Design. Chess skill was analyzed as a between-subject factor, with nine subjects at each skill level. Within each skill level, the memory task problems were arranged in a 9 by 9 GrecoLatin square counterbalancing test positions and presentation mode. There were nine chess positions to be remembered. Each chess position involved 24 pieces and pawns and had been taken from a game between chess masters (Chernev, 1960).

Procedure. The subjects were told that chess positions would be exposed briefly on the glass screen and that the display would change several times during each trial. Each was then instructed to attend carefully to the screen during each trial and to start reconstructing the position only after the stimulus display had terminated. Each chess position was presented in a series of six slides. Each slide appeared for $2 \mathrm{sec}$, with a time gap between successive slides of less than $2 \mathrm{msec}$. One trial (i.e., six slides) took $12 \mathrm{sec}$.

After each 12-sec presentation, the subject was given a maximum of 2 min to reconstruct the position on a chess set which had been placed on a table in the IAC booth for that purpose. The experimenter entered the testing booth after $30 \mathrm{sec}$ of the recall period and recorded the subject's piece placements. Each subject viewed and recalled nine chess positions.

The primary independent variable in the memory task was the mode in which each six-slide display was constructed. In one mode, the chess position grew sequentially by meaningful chunks. That is, the first slide would show three to six pieces, such as a castled-king position. The second slide would show this same meaningful chunk plus an additional chunk, such as a pawn chain. The third slide would contain the first two chunks plus a third chunk. The entire board position appeared for only $2 \mathrm{sec}$ on the final slide of the six-slide sequence. Our definition of a meaningful chunk was based on the five relationships discussed by Chase and Simon (1973): attack at short distance, defense, proximity, piece color, and piece type. A second presentation mode also involved the sequential presentation of cumulative segments of the board except the board developed by columns. The board "grew" four pieces at a time starting with column 1 (white's queen rook file) and proceeding to columns $3,5,7,2,4,6,8$, in that order. To an experienced chess player, this order of board development is clearly nonmeaningful. The entire board appeared only on the final slide. The third presentation mode involved the presentation of the
Table 1

Number of Pieces Placed Correctly in Experiment II When a Meaningful Chess Position was Presented in Its Entirety or Sequentially by Columns or Meaningful Chunks

\begin{tabular}{lccc}
\hline \multicolumn{1}{c}{ Skill Level } & $\begin{array}{c}\text { Entire } \\
\text { Board }\end{array}$ & $\begin{array}{c}\text { Meaningful } \\
\text { Chunks }\end{array}$ & Columns \\
\hline High (Class A) & 16.1 & 17.5 & 13.6 \\
Medium (Class C) & 15.3 & 16.0 & 12.4 \\
Low (novice) & 6.9 & 7.6 & 6.1 \\
\hline
\end{tabular}

entire board position on each of the six slides. Each subject was shown three series of slides in each of the three presentation modes.

\section{Results}

Performance on the memory task was scored by counting the number of pieces placed correctly. These data are summarized in Table 1. Skill level had a major impact on recall, with the high (Class A), intermediate (Class $\mathrm{C}$ ), and low (novice) skill levels remembering $15.73,14.57,6.88$ pieces, respectively. These betweensubject differences were statistically significant $[F(2,16)$ $=39.80, \mathrm{p}<.001]$. The standard error of these means was .76 pieces.

The Mode of Presentation also had a major effect on recall performance, with the meaningful chunk procedure averaging 13.72 pieces, the entire procedure averaging 12.77 pieces, and the columns procedure averaging 10.69 pieces. These within-subject differences were also significant $[F(2,192)=15.09, p<.001]$. The standard error of these means was .40 pieces.

The data analysis also indicated that performance deteriorated across trials. Across the first, second, and third three-trial blocks, the subjects recalled 13.79 , 12.09 , and 11.30 peices $[F(2,192)=10.25, p<.001]$. No other main effects or interactions were statistically significant.

\section{Discussion}

The second experiment demonstrated that chess skill is correlated with recall performance for meaningful chess positions whether they are presented as a whole or presented sequentially by columns or by meaningful chunks. In each condition, the high- and medium-skill subjects showed much better recall than the novices.

A second finding was that the mode of presentation had an important effect on performance. Best recall occurred when the pieces were presented sequentially by meaningful chunks (13.7 pieces). Recall performance in the entire board condition was slightly lower $(12.8$ pieces), while performance with sequential presentation by columns was poorest (10.7 pieces). These results are consistent with Charness' (Note 1) results, in which chunks were manipulated by varying the sequence in which verbal labels were given for chess pieces. The results of both experiments strongly support the chunking hypothesis as proposed by Chase and Simon (1973).

The absence of an interaction between chess skill and presentation mode indicates that both strong and 
weak players "chunk" material when faced with this recall task. The strong chess players, however, seem to be more proficient in finding and effectively labeling these chunks.

Finally, the results indicated that performance deteriorated throughout the nine recall trials. This effect suggests that there is considerable proactive interference on the later trials, because one might expect, with a novel task such as this, that there would be some improvement in performance as the subjects became more familiar with the task requirements.

\section{EXPERIMENT III}

Chase and Simon (1973) have suggested that skilled chess players perform better than unskilled players in the recall task because they perceive a meaningful position in terms of larger perceptual "chunks." Although their memory capacity is similar to that of novice players, the masters can remember more pieces because each of the retained chunks is larger. Their data from middle-game positions indicated that a chess master averaged 7.7 chunks per position of 2.4 pieces each, while a beginner averaged 5.3 chunks per position of 1.8 pieces each. Apparently, their master recognized more chunks and bigger chunks. In addition to their chunking hypothesis which seems well supported by their data and our own data (Experiment II), Chase and Simon (1973) postulated a limited-capacity (six- to eight-chunk) short-term memory which was used to store the information between presentation and recall. They proposed that the defining characteristic for each chess "chunk" is stored in long-term memory and that humans perform the chess memory task by recognizing familiar chunks, storing a label, not necessarily verbal, for each chunk in short-term memory, and then recalling the individual pieces by decoding each label during the test period. Because skilled chess players have many more familiar chess patterns in long-term memory, they recognize more chunks and bigger chunks, and thereby have a more efficient labeling system for the limited capacity of short-term memory.

This model matches conventional notions of shortterm memory capacity (seven \pm two chunks) and fits their chunking data quite well. Since the memory task has traditionally involved only a 5 -sec presentation of each chess position, Chase and Simon (1973) assumed that recall performance must depend on the shortterm memory store. Storing information in long-term memory is thought to require about 5 to $10 \mathrm{sec} / \mathrm{chunk}$ (Simon, 1974).

This analysis has been questioned, however, by recent data. Charness (Note 1 ) repeated the basic chess memory study using the Brown-Peterson paradigm (Brown, 1958; Peterson \& Peterson, 1959). He inter polated $30 \mathrm{sec}$ of additional processing (computing a running sum of random digits, mentally rotating ar then copying abstract symbols, etc.) between stimul presentation and recall. Charness' (Note 1) data inc cated that these interpolated activities had little, if an: effect on recall for the chess positions. These resul strongly question Chase and Simon's (1973) idea th: the chess information is stored in short-term memor. Numerous investigators (e.g., Keppel \& Underwoor 1962; Murdock, 1961; Peterson \& Peterson, 195 have demonstrated that interpolated processing task tend to interfere with information stored in shor term memory.

The Chase and Simon (1973) model can be salvage if one assumes that the chess information is stored $i$ a context-specific format and will not be "disturbed by unrelated information. Although this revision is volves a major change in the model, it retains the e: sential features of the original idea. This extension $c$ their model seems logical given evidence (e.g., Ternes $b$ Yuille, 1972) for separate short-term stores for verbc and visual information. Our third experiment provide a further examination of the notion that the ches information is stored in a limited-capacity short-terr. memory.

\section{Method}

Subjects. The subjects were 20 male volunteers from th Northwestern University chess club ranging in age from 17 to 3 : years. Each subject had a USCF rating between 1,500 and 2,100

Apparatus. The equipment and testing environment wer identical to that used in the first and second experiments. Th stimuli were $35-\mathrm{mm}$ photographic slides of chess positions whicl had appeared as diagrams in a book on tournament chess games Each stimulus was a quiet middle-game position with 23 or 2 c pieces on the board.

Procedure. The subjects arrived at the laboratory individ ualiy, according to a prearranged appointment schedule. Eacl subject was introduced to the IAC booth and instructed to attend to the ground-glass screen. He was informed that one o two chess positions would be displayed and that a three-digi number would then appear. When the number was presented he was to start counting backward by sevens in a loud voice He continued counting backward by sevens until he was in structed to recall, by reconstruction on a chess board, either the single position or one of the two chess positions which had beer displayed.

Each subject was given 12 memory trials. One-third of the trials (four) consisted of a single chess position, followed by counting, followed by a recall test. One-third of the trials (four) consisted of two chess positions shown in sequence, followed by counting, followed by recall of the first chess diagram. Onethird of the trials (four) consisted of two chess positions shown in sequence, followed by counting, followed by recall of the second chess diagram. One of each of these three trial types occurred during Trials 1-3, 4-6, 7-9, and 10-12. Half of the trials in each condition involved a recall test after $3 \mathrm{sec}$ and the other half had a recall test after $30 \mathrm{sec}$. In each condition, one recall test at each interval was given during Trials $1-6$ and the second was given during Trials 7-12. Each chess diagram was presented for $8 \mathrm{sec}$, and there was a 1-sec delay between slides when two positions were presented in sequence. During each recall period. the subject was given $90 \mathrm{sec}$ to reconstruct the designated position from memory on a chess board. After $30 \mathrm{sec}$, the experi- 
Table 2

Number of Pieces Placed Correctly in Experiment III as a Function of Presentation Condition and Time of Recall

\begin{tabular}{ccc}
\hline & \multicolumn{2}{c}{$\begin{array}{c}\text { Duration of Interpolated } \\
\text { Activity Between Presen- } \\
\text { tation and Recall }\end{array}$} \\
\cline { 2 - 3 } Condition & $3 \mathrm{Sec}$ & $30 \mathrm{Sec}$ \\
\hline A only, Recall A & 13.1 & 11.6 \\
A, then B, Recall B & 10.6 & 10.4 \\
A, then B, Recall A & 9.9 & 8.5 \\
\hline
\end{tabular}

menter entered the booth and started recording the subjects' placements.

\section{Results}

Recall performance was measured in terms of the number of pieces placed correctly in each condition. These data are presented in Table 2 . Table 2 indicates that recall was best when only one chess diagram had been presented ( 12.3 pieces). When two diagrams were presented, recall of the second diagram ( 10.5 pieces) was better than recall of the first diagram ( 9.2 pieces). These differences were highly reliable $[F(2,209)=13.15$, $p<.001]$. A subsequent Neuman-Keuls test $(\alpha=.05)$ indicated that each condition was reliably different from the others.

Table 2 also indicates that recall at $3 \mathrm{sec}(11.2$ pieces) was slightly better than recall at $30 \mathrm{sec}(10.2$ pieces). This difference was reliable $[F(1,209)=4.38$, $p<.05]$. The interaction between recall interval and the three conditions was not significant $[F(2,209)=.82]$. The standard error of the means in Table 2 is .43 pieces. The effect of recall interval depended on the stage of testing, with little or no difference between 3 and $30 \mathrm{sec}$ during the first six trials (11.0 and 11.1 pieces, respectively) and poorer performance at the longer interval during the last six trials (11.5 and 9.3 pieces, respectively) $[F(1,209)=5.41, p<.025]$. The standard error of these means was .35 pieces. No other main effect or interactions with this dependent measure were significant.

An analysis was also made of the number of intrusion errors in the two conditions in which two different diagrams were presented in sequence. An intrusion error was defined as an error of commission in which the incorrect placement would have been correct for the nonrecalled diagram. The intrusion errors for the two conditions as a function of stage of testing are depicted in Table 3. During the first six trials, intrusion errors were most prominent when the subject recalled the second diagram. During the last six trials, intrusion errors were equally likely in both recall conditions. This interaction was significant $[F(1,133)=5.08$, $\mathrm{p}<.05]$. The standard error of the means in Table 3 is .18 pieces. No other effects or interactions with this dependent measure were significant. The overall level of intrusion errors was $21 \%$ of the number of pieces placed correctly in the two-diagram conditions.

\section{Discussion}

The results of our third experiment do not support the notion that memory for chess positions in the classic de Groot (1965) paradigm is stored in a limitedcapacity short-term memory, as postulated by Chase and Simon (1973). First of all, our data replicate Charness' (Note 1) finding that interpolated processing (counting backward by sevens in our study) has little detrimental effect on recall performance. There was essentially no difference between recall after $3 \mathrm{sec}$ and recall after $30 \mathrm{sec}$ during the first six trials.

Second, Chase and Simon's (1973) model suggests that one middle-game position (i.e., 24 pieces) essentially saturates short-term memory. Reasoning on this basis, one would predict that two diagrams would over-saturate the capacity of short-term memory and that therefore, recall to each one would be about half as good as recall to a single diagram. The data presented in Table 2 are clearly inconsistent with this prediction. Recall performance in the two-diagram conditions was almost as good as in the single-diagram condition.

Third, a detailed comparison of the results depicted in Table 2 with Chase and Simon's (1973) results cast doubt on the notion that the site of storage is limited to seven or eight chunks of information. Our subjects recalled 12.3 pieces on the average in the single-diagram condition and 9.8 pieces in the two-diagram condition. According to the Chase and Simon model, our subjects would have to have labels in short-term memory representing 19.6 pieces in order to recall 9.8 pieces when they had no prior knowledge of which diagram would be tested. Chase and Simon's (1973) data indicated that their Class A player (equivalent to our best subjects) employed chunks of Size 2.6, 2.5, 1.8, and 1.6 as his first four chunks on the memory tasks. If our subjects could have stored four chunks in STM for the first diagram and four chunks in STM for the second diagram (i.e., eight chunks total) and their chunk size was as large as that for Chase and Simon's Class A player, one could project an average recall score on the two-diagram task of 8.5 pieces. This is less than the 9.8 pieces we observed despite the fact that our subjects, on the average, were below the Class $A$ level and that Chase and Simon's Class A player averaged about six chunks per trial rather than eight. These considerations also question the limited-capacity notion.

Fourth, even if the chess information is stored in a context-specific memory site, this should not prevent displacement of prior information by a second input when both inputs are chess related. The relatively

Table 3

Number of Intrusion Errors in Experiment III as a Function of Presentation Condition and Stage of Training

\begin{tabular}{ccc}
\hline Condition & Trials 1-6 & Trials 7-12 \\
\hline A, then B, Recall B & 2.7 & 1.9 \\
A, then B, Recall A & 1.6 & 2.2 \\
\hline
\end{tabular}


small difference observed between recall of the first diagram (9.2 pieces) and recall of the second diagram (10.5 pieces) suggests that the second input did not substantially displace the information from the first input. In fact, there is very little evidence of displacement at all, as one might expect for a limited-capacity store. Losses of information seem to be related more to interference effects (i.e., retrieval problems) than to displacement per se. There was a relatively high proportion of instrusion errors (21\%). During the first six trials, these intrusions were primarily proactive (Table 3); items from the first diagram were identified as being from the second diagram. A displacement effect should have produced the opposite pattern of intrusions. Moreover, the interaction between stage of testing and recall interval indicates that the small drop in performance between 3 and $30 \mathrm{sec}$ which was observed occurred only after the subjects had experienced 10 prior diagrams. This clearly suggests that the small decrease in recall scores is related to proactive interference rather than to displacement.

In summary, the data from our third experiment strongly question the notion that memory for briefly presented chess diagrams is held in a limited-capacity short-term memory. These data even appear to exclude a modified form of the Chase and Simon (1973) model which postulates a context-specific short-term memory store. The data necessitate a revision of the model to assume a short-term memory which holds either larger chunks or more chunks or to assume that the chess information is transmitted directly to long-term memory during the initial brief presentation.

\section{GENERAL DISCUSSION}

The present studies extend previous findings (Chase \& Simon, 1973; de Groot, 1965) indicating that memory for briefly exposed chess positions is a function of chess skill and that this relationship is critically dependent upon the amount of chess-specific information contained in the stimulus display. Additionally, the notion that skilled players cope with the relatively complex visual inputs by "chunking" pieces into familiar groupings (Chase \& Simon, 1973; Charness, Note 1) was supported by directly manipulating chunking. The development of a chess position in terms of a sequence of familiar piece groupings leads to much better recall than a similar sequential development in terms of unusual piece groupings. Thus, our first two experiments strongly confirm the prior observations that skilled chess players perform well in the chess memory task because of chess-specific knowledge rather than extraordinary memory capacity and that this superior performance derives from a learned perceptual facility for grouping chess pieces into meaningful chunks.

The results of the third experiment, however, strongly question the notion that recall for briefly ex- posed chess positions is mediated by storing these meaningful chunks in a limited-capacity short-term memory. Charness (Note 1) has previously demonstrated that interpolated processing between presentation and recall delays the rate of recall of chess information but does not reduce the accuracy of recall. His data from the Brown-Peterson paradigm suggest that the chess positions are not being stored in short-term memory in the same way that verbal material seems to be stored (Keppel \& Underwood, 1962; Murdock, 1961; Peterson \& Peterson, 1959). Our data replicated this finding and, in addition, provided evidence that the amount of material being stored was more than could be expected for the traditional limited-capacity model of shortterm memory. Finally, the data from our third experiment suggested that recall was limited by proactive interference rather than by the displacement of material from short-term memory by more recent items. These findings cast considerable doubt on the memory assump. tions in the Chase and Simon (1973) model.

Charness' (Note 1) data in conjunction with our results seem to imply that the information imparted by the chess diagrams is processed relatively deeply in a brief period of time and is then passed directly to a long-term store. Recent evidence indicating that pictorial information is processed very rapidly to an abstract level of meaning (Nelson, Metzler, \& Reed, 1974; Potter, 1975) is consistent with this interpretation. The research by Shaffer and Shiffrin (1972) on recognition of pictures suggests a similar mechanism in that environment. These results seem most consistent with Craik and Lockhart's (1972) view that recall ability depends on the depth of processing. In our situation, the more skilled players not only identify more chunks and bigger chunks, but also discover more semantic relations among the pieces and among the chunks. This deeper level of processing may in fact be the key to better retention.

\section{REFERENCE NOTE}

1. Charness, N. H. Memory for chess positions: The effects of interference and input modality. Unpublished $\mathrm{PhD}$ dissertation. Carnegie-Mellon University, 1974.

\section{REFERENCES}

Brown, J. Some tests of the decay theory of immediate memory. Quarterly Journal of Experimental Psychology, $1958,10,12-21$

Chase, W. G.. \& Simon. H. A. Perception in chess. Cognitive Psychology, 1973, 4, 55-81.

Chernev, I. Combinations: The heart of chess. New York: Crowell, 1960.

Craik, F. I. M., \& LockhaRT, R. S. Levels of processing: A framework for memory research. Joumal of Verbal Learning and Verbal Behavior, 1972, 11, 671-684.

DE GROOT, A. Thought and choice in chess. The Hague: Mouton, 1965.

Keppel, G., \& Underwood, B. J. Proactive inhibition in short-term retention of single items. Journal of Verbal 
Learning and Verbal Behavior, 1902, 1, 153-161.

Murdock. B. B. The retention of individual items. Journal of Experimental Psychology, 1961, 62, 618-625.

MyERS. J. L. Fundamentals of Experimental Design. Boston: Allyn \& Bacon, 1972.

Nelson. T. O.. Metzler, J., \& Reed, D. A. Role of details in the long-term recognition of pictures and verbal descriptions. Journal of Experimental Psychology, 1974. 102. $184-186$

Peterson, L. R., \& Peterson, M. J. Short-term retention of individual verbal items. Joumal of Experimental Psychology, 1959, 58, 193-198.

Potter, M. C. Meaning in visual search. Science, 1975, 187, 965-966.
Shaffer, D., \& Shiffrin, R. M. Rehearsal and storage of visual information. Journal of Experimental Psychology, $1972,92,292-296$.

Simon, H. A. How big is a chunk? Science, 1974, 183, 482-488.

Ternes, W., \& Yuille, J. C. Words and pictures in an STM task. Journal of Experimental Psychology, 1972, 96. 78-86.

(Received for publication October 21, 1975; revision accepted January 21,1976 .) 